\title{
Insomnia in Emotional Labor: Its Role in Autonomic Nervous System Regulation
}

\author{
Boram Chae ${ }^{1}$, June Kang ${ }^{2} \bowtie$, Cheolmin Shin ${ }^{1}$, Young-Hoon $\mathrm{Ko}^{1}$, and Ho-Kyoung Yoon ${ }^{1 凶}$ \\ ${ }^{1}$ Department of Psychiatry, Korea University Ansan Hospital, Ansan, Republic of Korea \\ ${ }^{2}$ Department of Brain and Cognitive Engineering, Korea University, Seoul Republic of Korea
}

Objective The relation between female emotional laborers' sleep quality and autonomic nervous system activity was investigated.

Methods Thirty-three subjects' heart rate variability (HRV) data and results of self-reported scale on sleep, depression, anxiety and suicidality, were collected. Subjects were classified into good sleeper (GS) and poor sleeper (PS) groups relying on sleep quality. Changes of HRV between working time and resting time in each group were compared.

Results The PS group showed significantly lower difference in root mean square of successive differences (RMSSD), percentage of successive normal-to-normal intervals that differ by more than $50 \mathrm{~ms}$ (pNN50), and natural logarithm high-frequency (LnHF) when they were working as compared to when they were resting, which means decreased function of the parasympathetic nervous system (PNS). Repeated measures analysis of covariance showed that the group effect was significant only for LnHF, with score of depression scale as a covariate.

Conclusion Female emotional laborers who complain of sleep difficulty may have decreased function of the PNS. Therefore, good sleep quality is essential for maintaining and promoting mental and physical health of women engage in emotional labor.

Psychiatry Investig 2021;18(9):889-894

Keywords HRV; Autonomic nervous system; Emotional labor; Sleep; Parasympathetic nervous system.

\section{INTRODUCTION}

As women and girls are expected to be much more emotionally aware and better at providing emotional support than men and boys, the proportion of women is higher in work related to emotional labor. The survey on emotional laborers' mental health conducted by Ansan Community Mental Health Center in 2018 reported that $84.5 \%$ of the respondents were female. ${ }^{1}$

Emotional labor refers to the form of emotional regulation in which individuals adjust their posture and facial expressions to meet organizational requirements. ${ }^{2}$ To meet emotional job

\footnotetext{
Received: April 8, 2021 Revised: July 11, 2021 Accepted: July 18, 2021

$\triangle$ Correspondence: June Kang, $\mathrm{PhD}$

Department of Brain and Cognitive Engineering, Korea University, 145 Anamro, Seongbuk-gu, Seoul 02841, Republic of Korea

Tel: +82-2-6401-0199, Fax: +82-2-6280-1626

E-mail: June.kang@empathy-research.org

$\triangle$ Correspondence: Ho-Kyoung Yoon, $\mathrm{MD}, \mathrm{PhD}$

Department of Psychiatry, Korea University Ansan Hospital, 123 Jeokgeum-ro, Danwon-gu, Ansan 15355, Republic of Korea

Tel: +82-31-412-5140, Fax: +82-31-412-5132, E-mail: hkhkgogo@korea.ac.kr

(a) This is an Open Access article distributed under the terms of the Creative Commons Attribution Non-Commercial License (https://creativecommons.org/licenses/bync/4.0) which permits unrestricted non-commercial use, distribution, and reproduction in any medium, provided the original work is properly cited.
}

demands, workers need to exert self-control, especially when they have to display emotions that they do not genuinely feel. ${ }^{3}$ According to the survey, "Right to health of retail service and sales workers" published by the National Human Rights Commission of Korea in 2015, 61\% of emotional laborers experienced verbal or physical violence during the last year. In addition, $96 \%$ have tried to hide or distort their real emotions in their contact with clients or customers. ${ }^{4}$ This emotional dissonance requires significant self-control and depletes limited regulatory resources, leading to exhaustion and burnout. ${ }^{5}$

Emotional dissonance can be reflected by a change in autonomic nervous system (ANS) activities assessed in terms of heart rate variability (HRV), ${ }^{6}$ the fluctuation in the time intervals between adjacent heartbeats. A healthy heart's beat-to-beat fluctuations are not like a metronome and are better described as mathematical chaos. ${ }^{7}$ The fluctuations provide the flexibility to rapidly cope with an uncertain and changing environment. However, when an organism exhausts all its resources, the acquired adaptation or resistance is lost and is reflected as HRV.

Sleep difficulty has been associated with a variety of physical and mental health-related outcomes. ${ }^{8}$ Owing to the restorative function of sleep, good sleep stabilizes the cerebral meta- 
bolic rate ${ }^{9}$ and ensures adequate resources or energy supply to the prefrontal cortex whose structures are important for executive self-control. ${ }^{10}$ Good sleep facilitates self-control in functioning during the following day. ${ }^{6}$ Conversely, poor sleep prevents restorative processes and further induces chemical and structural central nervous system cellular injury, leading to dysfunction of prefrontal regions of the brain cortex and deficits in self-control. ${ }^{11}$ Therefore, poor sleep has the potential to impair emotional regulation as a form of self-control.

We hypothesized that sleep difficulty in emotional laborers is associated with impairment of emotional regulation and burnout, leading to ANS dysregulation. Discovering the associations between insomnia and ANS dysregulation can provide evidence for emotional regulation difficulty in emotional laborers.

\section{METHODS}

Thirty-three female customer service representatives were recruited from 2 reservation call centers at Korea University Ansan Hospital and Anam Hospital. Participation in the research was entirely voluntary, and confidentiality was guaranteed.

The subjects were requested to provide personal information such as age, education level completed, marital state, living status, and tenure. In addition, the participants were asked to fill out self-reported questionnaires that assessed their psychologi$\mathrm{cal}$ state. The participants were required to wear a wristband during working time and resting time on 2 workdays.

The questionnaires included the Athens Insomnia Scale-8 (AIS-8), Center for Epidemiological Studies Depression Scale (CES-D), Generalized Anxiety Disorder 7-item (GAD-7), and Suicide Behaviors Questionnaire-Revised (SBQ-R). The AIS-8 measures the intensity of insomnia. The AIS- 8 contains 8 items and is scored on a 4-point numerical rating scale: $0=$ no problem, $1=$ minor problem, $2=$ marked problem, and $3=$ very serious problem. Total scores range from 0 to 24 , with a higher score indicating poorer sleep. A cut-off score of 8 was used in the present study. ${ }^{12,13}$ The Cronbach's alpha of AIS- 8 was 0.80 . The CES-D is commonly used to measure depressive symptoms; response options range from 0 to 3 for each item, and scores range from 0 to 60 with high scores indicating stronger depressive symptoms. ${ }^{14}$ The cut-off point was 20 , and the Cronbach's alpha was 0.93 . The GAD-7 has 7 items, some of which are included in the Diagnostic and Statistical Manual's (DSM-V) criteria for GAD to identify probable cases of GAD while also measuring anxiety symptom severity. ${ }^{15}$ With a threshold score of 10 , the Cronbach's alpha was 0.87 . The SBQ- R is designed to identify risk factors for suicide. The cut-off score is 7 for adults from the general population (sensitivity of $93 \%$, specificity of $81 \%) .{ }^{16}$ The Cronbach's alpha of SBQ-R was 0.75 .

HRV data for this study were obtained using the $\mathrm{E} 4$ wristband
(Empatica, Boston, MA, USA) which is validated research instrument for heart rate (HR) and HRV. ${ }^{17,18}$ The $\mathrm{E} 4$ wristband is a wearable and non-invasive device that allows real-time physiological data collection. This device is equipped with a photoplethysmogram sensor to measure blood volume pulse, from which HR and interbeat intervals (IBI) are derived. Several HRV time domain measures can be calculated from the IBI data such as standard deviation of normal-to-normal (SDNN), root mean square of successive differences (RMSSD), the number of successive normal-to-normal interval pairs that differ by more than $50 \mathrm{~ms}$ (NN50), and the percentage of successive normal-tonormal intervals that differ by more than $50 \mathrm{~ms}$ (pNN50). Both sympathetic nervous system (SNS) and parasympathetic nervous system (PNS) activity contribute to SDNN. ${ }^{19}$ The RMSSD has previously been shown to be a valid index of PNS activity. ${ }^{20}$ The pNN50 is also closely correlated with PNS activity. ${ }^{19}$ HRV frequency domain measures can also be produced based on the IBI series such as low-frequency (LF) band (0.04-0.15 $\mathrm{Hz}$ ) and high-frequency (HF) band $(0.15-0.40 \mathrm{~Hz})$. Autoregressive spectral analysis and component wave analysis were applied to the IBI for extraction of LF and HF bands. The LF band includes both PNS and SNS influences and is highly correlated with SDNN. HF power reflects PNS activity. ${ }^{21}$ Natural logarithm LF (LnLF) and natural logarithm HF (LnHF) can be calculated from the data.

\section{Statistical analyses}

Participants were respectively classified into poor sleeper (PS) and good sleeper (GS) groups based on the AIS-8 cut-off score. The general characteristics of the participants in PS and GS groups were compared using Mann-Whitney U-test or chisquare test. HRV variables between working time and resting time were compared within each group. Comparisons were obtained using the Wilcoxon signed rank test. Lastly, we compared the value of the HRV parameters between PS and GS. We examined the effect of sleep difficulty on the differences of the HRV parameters between working time and resting time by performing an analysis of covariance (ANCOVA) with repeated measures. The between-subjects factor was group (GS vs. PS) and the within-subjects factor was time (work vs. rest). The CES-D score was used as the covariates because this variable has been shown to be related with the AIS score. All data were statistically analyzed by SPSS for Windows version 25 (IBM Corp., Armonk, NY, USA).

\section{Ethical issues}

The study protocol was reviewed and approved by the Institutional Review Board from Korea University (KUIRB-20190168-01). 


\section{RESULTS}

The present study included 33 female subjects. The mean \pm standard deviation (SD) age was $35.52 \pm 7.11$ years (range 2247 years). Among the subjects, $45.4 \%(\mathrm{n}=15)$ were married, and $54.5 \%(n=18)$ were singles. The majority $(87.8 \%[n=29])$ of the subjects were living with someone else, whereas $12.1 \%$ $(n=4)$ were living by themselves. The mean duration of employment was 4 years $(\mathrm{SD}=4.93)$. Mean scores and $\mathrm{SDs}$ of each psychological scale are summarized in Table 1.

Subjects were classified based on the AIS- 8 cut-off score into good sleeper (GS) and poor sleeper (PS) groups. The GS group consisted of 17 subjects (51.5\%) and the PS group of 16 subjects (48.5\%). The average AIS-8 scores were 4.24 \pm 4.92 in the GS group and $9.68 \pm 1.62$ in the PS group.

Because the sample size in the GS group was small $(n=17)$, the Wilcoxon signed rank test was used to compare HRV parameters between working time and resting time in the GS group. $\mathrm{SDNN}$ (difference $=-2.06, \mathrm{p}=0.039$ ), NN50 (difference=
-3.62, $\mathrm{p}<0.001$ ), and LnLF (difference $=-3.00, \mathrm{p}=0.003$ ) were significantly different. No significant difference was observed in RMSSD (difference $=-0.21, \mathrm{p}=0.831$ ), pNN50 (difference= $-0.45, \mathrm{p}=0.653)$ and LnHF power (difference $=-1.40, \mathrm{p}=0.162$ ) (Table 2).

As was the case for the GS group, Wilcoxon signed rank test was used to compare HRV parameters between working time and resting time for the PS group because of the small sample size $(n=16)$. All HRV parameters turned out to be significantly different for the PS group: $\operatorname{SDNN}$ (difference $=-3.26, \mathrm{p}=0.001$ ), RMSSD (difference $=-3.05, \mathrm{p}=0.002$ ), NN50 (difference $=-3.52$, $\mathrm{p}<0.001$ ), pNN50 (difference $=-2.84, \mathrm{p}=0.004)$, LnLF (difference $=-2.74, \mathrm{p}=0.006)$ and LnHF (difference $=-3.36, \mathrm{p}=0.001$ ) (Table 3).

No significant differences were observed in age, marital status, living status, education duration, tenure, and SBQ-R scores between PS and GS groups. Conversely, significant differences were observed in CES-D and GAD-7 between both groups (Table 1). Pearson's correlation was used to investigate whether

Table 1. Sociodemographic and psychological characteristics of subjects

\begin{tabular}{|c|c|c|c|c|c|}
\hline \multirow{2}{*}{ Variables } & \multirow{2}{*}{ Categories } & \multirow{2}{*}{$\begin{array}{l}\text { Total }(\mathrm{N}=33) \\
\mathrm{N} \text { or } \mathrm{M} \pm \mathrm{SD}\end{array}$} & \multirow{2}{*}{$\begin{array}{c}\mathrm{GS}(\mathrm{N}=17) \\
\mathrm{N}(\%) \text { or } \mathrm{M} \pm \mathrm{SD}\end{array}$} & \multirow{2}{*}{$\begin{array}{c}\mathrm{PS}(\mathrm{N}=16) \\
\mathrm{N}(\%) \text { or } \mathrm{M} \pm \mathrm{SD}\end{array}$} & \multirow{2}{*}{$\chi^{2}$ or $\mathrm{u}$} \\
\hline & & & & & \\
\hline Age & & $35.52 \pm 7.11$ & $37.82 \pm 6.79$ & $32.47 \pm 6.58$ & $\mathrm{u}=89.5$ \\
\hline \multirow[t]{2}{*}{ Marital status } & Unmarried & $18(54.5)$ & $8(47.1)$ & $10(62.5)$ & \multirow[t]{2}{*}{$\chi^{2}=0.79$} \\
\hline & Married & $15(45.4)$ & $9(52.9)$ & $6(37.5)$ & \\
\hline \multirow[t]{2}{*}{ Living status } & Live alone & $4(12.1)$ & $1(5.9)$ & $3(18.8)$ & \multirow[t]{2}{*}{$\chi^{2}=1.28$} \\
\hline & Live with others & $29(87.8)$ & $16(94.1)$ & $13(81.3)$ & \\
\hline Education duration (yr) & & $14.18 \pm 1.53$ & $14.59 \pm 1.54$ & $13.73 \pm 1.49$ & $\mathrm{u}=94.5$ \\
\hline Tenure (yr) & & $4.00 \pm 4.93$ & $4.77 \pm 4.92$ & $3.35 \pm 5.08$ & $\mathrm{u}=89.0$ \\
\hline AIS-8 & & $6.88 \pm 3.41$ & $4.24 \pm 2.33$ & $9.80 \pm 1.61$ & $\mathrm{u}=0.00^{*}$ \\
\hline CES-D & & $12.24 \pm 10.67$ & $5.82 \pm 5.25$ & $19.06 \pm 10.61$ & $\mathrm{u}=35.5^{*}$ \\
\hline GAD-7 & & $4.09 \pm 3.76$ & $2.59 \pm 3.64$ & $5.80 \pm 3.21$ & $\mathrm{u}=46.0^{*}$ \\
\hline SBQ-R & & $3.61 \pm 1.89$ & $3.12 \pm 1.32$ & $4.20 \pm 2.34$ & $\mathrm{u}=101.50$ \\
\hline
\end{tabular}

${ }^{*} \mathrm{p}<0.05$. AIS-8, Athens insomnia scale-8; CES-D, Center for Epidemiological Studies-Depression Scale; GAD-7, Generalized Anxiety Disorder 7-item; SBQ-R, Suicide Behaviors Questionnaire-Revised; GS, good sleepers; PS, poor sleepers

Table 2. Changes in heart rate variability measures between work and rest in good sleepers

\begin{tabular}{llcccc}
\hline \multirow{2}{*}{ Domain } & Parameter & \multicolumn{2}{c}{ M \pm SD } & Diff (Work-Rest) & p \\
\cline { 3 - 5 } Time & SDNN (ms) & $87.38 \pm 17.82$ & $93.13 \pm 15.38$ & -2.06 & 0.039 \\
& RMSSD (ms) & $87.80 \pm 24.97$ & $87.62 \pm 26.29$ & -0.21 & 0.831 \\
& NN50 (beats) & $7,480.69 \pm 1929.31$ & $1,971.29 \pm 460.16$ & -3.62 & $<0.001$ \\
Frequency & pNN50 (\%) & $44.73 \pm 11.14$ & $45.64 \pm 10.87$ & -0.45 & 0.653 \\
& LnLF & $8.10 \pm 0.43$ & $8.35 \pm 0.25$ & -3.00 & 0.003 \\
\hline
\end{tabular}

SDNN, standard deviation of normal-to-normal; RMSSD, root mean square of successive differences; NN50, the number of successive normal-to-normal interval pairs that differ more than $50 \mathrm{msec}$; pNN50, percentage of successive normal-to-normal intervals that differ by more than $50 \mathrm{~ms}$; LnLF, natural logarithm low-frequency; LnHF, natural logarithm high-frequency 
these differences were related to sleep difficulty; GAD-7 ( $r=$ 0.450, $\mathrm{p}=0.01)$ and CES-D $(\mathrm{r}=0.639, \mathrm{p}<0.001)$ were found to be correlated with the AIS- 8 . Performing the partial correlation only revealed a relationship between AIS- 8 and CES-D $(\mathrm{r}=0.558, \mathrm{p}=0.001)$. A repeated measures ANCOVA was used to compare the differences of the HRV parameters between working time and resting time in the GS and PS groups. For LnHF, the group effect was significant with the CES-D score as a covariate $\left(\mathrm{F}_{1,30}=4.296 ; \mathrm{p}=0.047\right)$ (Table 4 and Figure 1$)$. The same repeated measures ANCOVA model performed for other

Table 3. Changes in heart rate variability measures between work and rest in poor sleepers

\begin{tabular}{|c|c|c|c|c|c|}
\hline \multirow{2}{*}{ Domain } & \multirow{2}{*}{ Parameter } & \multicolumn{2}{|c|}{$\mathrm{M} \pm \mathrm{SD}$} & \multirow{2}{*}{ Diff(Work-Rest) } & \multirow{2}{*}{$\mathrm{p}$} \\
\hline & & Work & Rest & & \\
\hline \multirow[t]{4}{*}{ Time } & SDNN & $85.58 \pm 21.87$ & $97.32 \pm 19.27$ & -3.26 & 0.001 \\
\hline & RMSSD & $90.31 \pm 26.84$ & $97.21 \pm 27.18$ & -3.05 & 0.002 \\
\hline & NN50 & $7,375.52 \pm 1,909.97$ & $2,151.00 \pm 494.13$ & -3.52 & $<0.001$ \\
\hline & pNN50 & $45.03 \pm 12.68$ & $49.93 \pm 12.42$ & -2.84 & 0.004 \\
\hline \multirow[t]{2}{*}{ Frequency } & $\operatorname{LnLF}$ & $8.00 \pm 0.48$ & $8.33 \pm 0.38$ & -2.74 & 0.006 \\
\hline & LnHF & $7.87 \pm 0.65$ & $8.11 \pm 0.56$ & -3.36 & 0.001 \\
\hline
\end{tabular}

SDNN, standard deviation of normal-to-normal; RMSSD, root mean square of successive differences; NN50, the number of successive normal-to-normal interval pairs that differ more than $50 \mathrm{msec}$; pNN50, percentage of successive normal-to-normal intervals that differ by more than 50 ms; LnLF, natural logarithm low-frequency; LnHF, natural logarithm high-frequency; Diff, difference

Table 4. Effect of the sleep quality on change of heart rate variability measures

\begin{tabular}{|c|c|c|c|c|c|}
\hline Source & Type III Sum of Squares & $\mathrm{df}$ & Mean SS & $\mathrm{F}$ & Sig \\
\hline SDNN & 593.83 & 1 & 593.83 & 9.16 & 0.005 \\
\hline SDNN*CES-D & 37.05 & 1 & 37.05 & 0.57 & 0.46 \\
\hline SDNN*group & 175.55 & 1 & 175.55 & 2.71 & 0.110 \\
\hline Residual & $1,944.12$ & 30 & 64.80 & & \\
\hline RMSSD & 39.98 & 1 & 39.98 & 0.84 & 0.37 \\
\hline RMSSD*CES-D & 1.61 & 1 & 1.61 & 0.03 & 0.86 \\
\hline RMSSD*group & 105.93 & 1 & 105.93 & 2.22 & 0.15 \\
\hline Residual & $1,432.31$ & 30 & 47.74 & & \\
\hline NN50 & $11,609,4891.26$ & 1 & $116,094,891.26$ & 96.45 & 0.00 \\
\hline NN50*CES-D & $1,543,690.16$ & 1 & $1,543,690.16$ & 1.28 & 0.27 \\
\hline NN50*group & $1,528,816.26$ & 1 & $1,528,816.26$ & 1.27 & 0.27 \\
\hline Residual & $36,111,184.31$ & 30 & $1,203,706.14$ & & \\
\hline pNN50 & 46.90 & 1 & 46.90 & 2.68 & 0.11 \\
\hline pNN50*CES-D & 0.28 & 1 & 0.28 & 0.02 & 0.90 \\
\hline pNN50*group & 43.34 & 1 & 43.34 & 2.47 & 0.13 \\
\hline Residual & 525.48 & 30 & 17.52 & & \\
\hline LnLF & 0.91 & 1 & 0.91 & 16.03 & 0.00 \\
\hline LnLF*CES-D & 0.15 & 1 & 0.15 & 2.58 & 0.12 \\
\hline LnLF*'group & 0.14 & 1 & 0.14 & 2.46 & 0.13 \\
\hline Residual & 1.70 & 30 & 0.06 & & \\
\hline LnHF & 0.19 & 1 & 0.19 & 8.46 & 0.01 \\
\hline LnHF*CES-D & 0.01 & 1 & 0.01 & 0.43 & 0.52 \\
\hline LnHF*'group & 0.10 & 1 & 0.10 & 4.30 & 0.05 \\
\hline Residual & 0.67 & 30 & 0.02 & & \\
\hline
\end{tabular}

SDNN, standard deviation of normal-to-normal; RMSSD, root mean square of successive differences; NN50, the number of successive normal-to-normal interval pairs that differ more than $50 \mathrm{msec}$; pNN50, percentage of successive normal-to-normal intervals that differ by more than $50 \mathrm{~ms}$; LnLF, natural logarithm low-frequency; LnHF, natural logarithm high-frequency; CES-D, Center for Epidemiological StudiesDepression Scale 


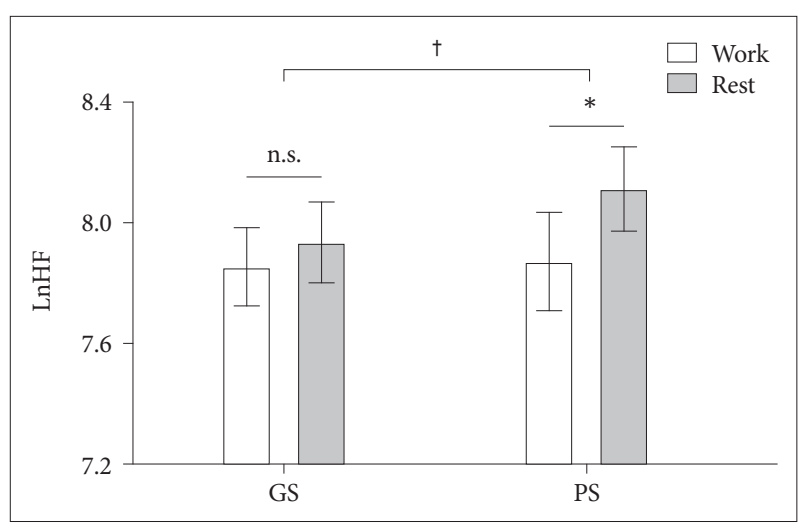

Figure 1. Comparison of LnHF in GS and PS groups during working time and resting. ${ }^{*} p<0.01 ;{ }^{\dagger} p<0.05$. GS, good sleepers; PS, poor sleepers; LnHF, natural logarithm high-frequency; n.s., not significant.

HRV parameters did not yield any significant interaction effects.

\section{DISCUSSION}

In growing recognition of the consequences of sleep problems, particularly for the working population, many researchers have suggested that sleep difficulty is associated with a variety of health problems ${ }^{22}$ including ANS dysregulation. The main finding of this study is that the value of the HRV parameters decreased during working time in female customer service representatives who had sleep difficulty. To the best of our knowledge, this is the first study to investigate the relationship between sleep difficulty and ANS dysregulation in female emotional laborers. The GS group did not show difference in RMS$\mathrm{SD}$, pNN50, and LnHF between working time and resting time. However, the PS group showed significantly lower RMSSD, pNN50, and LnHF when they were working as compared to when they were resting. Of note, after adjustment of the depression score, this difference persisted only in the LnHF. These results suggest that work-related reductions in HRV parameters in the PS group might be due to sleep difficulty-induced PNS dysfunction.

Sleep deprivation may cause changes in the neurotransmitter and neuroendocrine activity mediated by the autonomic sympatho-adrenal system and the hypothalamic-pituitary-adrenal axis, which is similar to a stress response. Hyperarousal has been hypothesized to contribute to the development, maintenance, and 24-hour systemic sequelae of insomnia. ${ }^{23}$ Physiological hyperarousal in insomnia patients has been investigated by measuring HRV. Several case-control studies have reported decreased HRV-related parasympathetic activity in insomnia patients, and these HRV impairments are in line with our findings. ${ }^{24,25}$ These results suggest altered parasympathetic function might be a putative mechanism for daytime dysfunction in insomnia.
People who suffer from sleep difficulty may have a poorly functioning PNS, which is associated with a variety of physical and mental health-related outcomes. ${ }^{8}$ Many researchers have reported that decreased vagal tone is observed as an independent predictor of new-onset hypertension, ${ }^{26}$ corroborating its importance in hypertension. ${ }^{27}$ Because the dominant role of the PNS is recovery and restoration, ${ }^{28}$ a chronically suppressed PNS may hinder the process of unwinding after work. Employees thereby fail to fully relax at home and struggle with sleep. This results in reduced metabolic activity in the prefrontal cortex whose structures are important for executive self-control. ${ }^{29} \mathrm{Be}-$ cause emotional labor requires significant control and attentive action, ${ }^{30}$ emotional laborers with sleep difficulty may feel too exhausted to exert self-control. Consequently, they may become more vulnerable to stress and fall into a vicious cycle. Given that PNS dysregulation is associated with sleep difficulty, managing the sleep difficulty should be prioritized to prevent chronic suppression of the PNS activity and protect workers from psychiatric and medical diseases.

The present study has several limitations. First, the sample size was not sufficiently large to ensure the validity of results. It might be the reason why there was no group-by-time interaction effect, although group comparison showed significant difference. Thus, another study with a larger cohort may be necessary to confirm the results. Second, this research was conducted with employees working at a call center who may not be true representatives of other occupational groups who work in the emotional labor field. Therefore, to validate the results, further studies that include various occupational groups are needed. Third, confounders such as cigarette smoking, alcohol consumption, body mass index, and habitual physical activity were not considered. In addition, the female hormone may have affected the study results. For more accurate results, adjustments should be made for various confounders. Lastly, the extent to which sleep difficulty precedes changes in PNS activity or whether sleep difficulty is a consequence of suppressed PNS activity remain unclear. The causality of the association between sleep disturbance and the PNS activity remains to be established.

The current study examined the relationship between sleep difficulty and ANS dysregulation in female emotional laborers. The HRV parameters associated PNS decreased during working time in female customer service representatives who had sleep difficulty. Sleep health care is essential to protect emotional laborers' mental health. If they have sleep problem, it can be helpful to guide them into mental health care programs such as cognitive-behavioral treatment for insomnia. ${ }^{31}$ This will be beneficial for their sleep health and lead to restoring of PNS. The relaxation therapy and mindfulness-based cognitive therapy are also expected to lower anxiety level and lessen sleep difficulty and emotional dissonance. ${ }^{32}$ 


\section{Availability of Data and Material}

The datasets generated or analyzed during the study are available from the corresponding author on reasonable request.

\section{Conflicts of Interest}

The authors have no potential conflicts of interest to disclose.

\section{Author Contributions}

Conceptualization: Ho-Kyoung Yoon. Data curation: June Kang, HoKyoung Yoon. Formal analysis: Boram Chae, June Kang, Ho-Kyoung Yoon. Investigation: June Kang, Ho-Kyoung Yoon. Methodology: June Kang, HoKyoung Yoon. Project administration: June Kang, Ho-Kyoung Yoon. Resources: June Kang, Ho-Kyoung Yoon. Supervision: Cheolmin Shin, YoungHoon Ko, Ho-Kyoung Yoon. Writing-original draft: Boram Chae, HoKyoung Yoon. Writing-review \& editing: Boram Chae, Ho-Kyoung Yoon.

\section{ORCID iDs}

$\begin{array}{ll}\text { Boram Chae } & \text { https://orcid.org/0000-0003-0737-2280 } \\ \text { June Kang } & \text { https://orcid.org/0000-0003-3625-0856 } \\ \text { Cheolmin Shin } & \text { https://orcid.org/0000-0002-8232-2921 } \\ \text { Young-Hoon Ko } & \text { https://orcid.org/0000-0002-5352-2158 } \\ \text { Ho-Kyoung Yoon } & \text { https://orcid.org/0000-0002-3302-0660 }\end{array}$

\section{Funding Statement}

This study was supported by the National Research Foundation of Korea (NRF) grant funded by the Korean government (MSIT) (No. 2020R1A2C 1008072), and the Korea University Grant.

\section{REFERENCES}

1. Lee J, Han C, Ko YH, Kang J, Byun Y, Son Y, et al. Emotional changes and protective factors of emotional workers in the public and private sector. Psychiatry Investig 2020;17:645-653.

2. Hochschild AR. The Managed Heart: Commercialization of Human Feeling. CA: University of California Press; 2012.

3. Zapf D, Holz M. On the positive and negative effects of emotion work in organizations. Eur J Work Organ Psychol 2006;15:1-28.

4. Kim JJ. Survey on Right to Health of Retail Service and Sales Workers. Seoul: National Human Rights Commission of the Republic of Korea; 2015.

5. Hagger MS, Wood C, Stiff C, Chatzisarantis NL. Ego depletion and the strength model of self-control: a meta-analysis. Psychol Bull 2010;136: 495-525.

6. Hjortskov N, Rissen D, Blangsted AK, Fallentin N, Lundberg U, Sogaard $\mathrm{K}$. The effect of mental stress on heart rate variability and blood pressure during computer work. Eur J Appl Physiol 2004;92:84-89.

7. Goldberger AL. Is the normal heartbeat chaotic or homeostatic. News Physiol Sci 1991;6:87-91

8. Nakata A, Haratani T, Takahashi M, Kawakami N, Arito H, Kobayashi F, et al. Job stress, social support, and prevalence of insomnia in a population of Japanese daytime workers. Soc Sci Med 2004;59:1719-1730.

9. Lenzi P, Zoccoli G, Walker AM, Franzini C. Cerebral blood flow regulation in REM sleep: a model for flow-metabolism coupling. Arch Ital Biol 1999;137:165-179.

10. Hofmann W, Schmeichel BJ, Baddeley AD. Executive functions and self-regulation. Trends Cogn Sci 2012;16:174-180.

11. Barnes CM, Ghumman S, Scott BA. Sleep and organizational citizenship behavior: the mediating role of job satisfaction. J Occup Health Psychol 2013;18:16-26.

12. Enomoto K, Adachi T, Yamada K, Inoue D, Nakanishi M, Nishigami T, et al. Reliability and validity of the Athens Insomnia Scale in chronic pain patients. J Pain Res 2018;11:793-801.
13. Chiang HL, Chen $\mathrm{HC}$, Bai $\mathrm{CH}$, Che HH, Lee MB, Lai SH, et al. A validation study of the Chinese version of the Athens insomnia scale. Taiwan J Psychiatry 2009;23:43-52.

14. Radloff LS. The CES-D scale: a self-report depression scale for research in the general population. Appl Psychol Meas 1977;1:385-401.

15. American Psychiatric Association. Diagnostic and statistical manual of mental disorders, Fifth edition. Arlington, VA: American Psychiatric Publishing; 2013.

16. Osman A, Bagge CL, Gutierrez PM, Konick LC, Kopper BA, Barrios FX. The Suicidal Behaviors Questionnaire-Revised (SBQ-R): validation with clinical and nonclinical samples. Assessment 2001;8:443-454.

17. Schuurmans AAT, de Looff P, Nijhof KS, Rosada C, Scholte RHJ, Popma A, et al. Validity of the empatica $\mathrm{E} 4$ wristband to measure heart rate variability (HRV) parameters: a comparison to electrocardiography (ECG). J Med Syst 2020;44:190.

18. Van Lier HG, Pieterse ME, Garde A, Postel MG, De Haan HA, Vollenbroek-Hutten MMR, et al. A standardized validity assessment protocol for physiological signals from wearable technology: methodological underpinnings and an application to the E4 biosensor. Behav Res Methods 2020;52:607-629.

19. Umetani K, Singer DH, McCraty R, Atkinson M. Twenty-four hour time domain heart rate variability and heart rate: relations to age and gender over nine decades. J Am College Cardiol 1998;31:593-601.

20. Camm AJ, Malik M, Bigger JT, Breithardt G, Cerutti S, Cohen RJ, et al. Heart rate variability - Standards of measurement, physiological interpretation, and clinical use. Circulation 1996;93:1043-1065.

21. Grossman P, Taylor EW. Toward understanding respiratory sinus arrhythmia: relations to cardiac vagal tone, evolution and biobehavioral functions. Biol Psychol 2007;74:263-285.

22. Knudsen HK, Ducharme LJ, Roman PM. Job stress and poor sleep quality: data from an American sample of full-time workers. Soc Sci Med 2007;64:1997-2007.

23. Riemann D, Spiegelhalder K, Feige B, Voderholzer U, Berger M, Perlis $\mathrm{M}$, et al. The hyperarousal model of insomnia: a review of the concept and its evidence. Sleep Med Rev 2010;14:19-31.

24. Bonnet MH, Arand DL. Heart rate variability in insomniacs and matched normal sleepers. Psychosom Med 1998;60:610-615.

25. Spiegelhalder K, Fuchs L, Ladwig J, Kyle SD, Nissen C, Voderholzer U, et al. Heart rate and heart rate variability in subjectively reported insomnia. J Sleep Res 2011;20:137-145.

26. Singh JP, Larson MG, Tsuji H, Evans JC, O’Donnell CJ, Levy D. Reduced heart rate variability and new-onset hypertension: insights into pathogenesis of hypertension: the Framingham Heart Study. Hypertension 1998;32:293-297.

27. Julius $S$. Autonomic nervous system dysregulation in human hypertension. Am J Cardiol 1991;67:3B-7B.

28. Carravieri A, Muller MS, Yoda K, Hayama S, Yamamoto M. Dominant parasympathetic modulation of heart rate and heart rate variability in a wild-caught seabird. Physiol Biochem Zool 2016;89:263-276.

29. Hare TA, Camerer CF, Rangel A. Self-control in decision-making involves modulation of the vmPFC valuation system. Science 2009;324: 646-648.

30. Morris JA, Feldman DC. The dimensions, antecedents, and consequences of emotional labor. Acad Manag Rev1996;21:986-1010.

31. Schutte-Rodin S, Broch L, Buysse D, Dorsey C, Sateia M. Clinical guideline for the evaluation and management of chronic insomnia in adults. J Clin Sleep Med 2008;4:487-504.

32. Jung YH, Ha TM, Oh CY, Lee US, Jang JH, Kim J, et al. The effects of an online mind-body training program on stress, coping strategies, emotional intelligence, resilience and psychological state. PLoS One 2016; 11:e0159841. 